\title{
Energy - Information Regularities of Increasing Productivity in Metalworking Machine Tools
}

\author{
Alexander P. Kuznetsov ${ }^{I}$ and Hans-Joachim Koriath ${ }^{2 *}$ \\ ${ }^{1}$ Moscow State Technological University "STANKIN", RU-127055, Moscow, Russia \\ ${ }^{2}$ Fraunhofer Institute for Machine Tools and Forming Technology IWU, DE-09126, Chemnitz, Germany
}

\begin{abstract}
Increasing productivity by higher cutting speed and achieving high precision of machined products at the same time is an important trend in the development of manufacturing technologies and metalworking machine tools. If the traditional method of exchangeable components in assemblies will lead to inefficient precise processes, the product costs will increase. The proposed energy-informational model considers a procedural system establishing the ratio of the cutting speed and the speed of physical processes in the machine tools. The energetic limit for the attainability of associated processes describes the allowable speed of materials processing by the machine tools. Stiffness of machine tool structures for highest precision manufacturing becomes comparable to work piece geometrical accuracy and manufacturing process tolerances. This paper gives several examples for admissible limits of increased productivity by increased process speeds in various manufacturing technologies for both traditional cutting and innovative methods.
\end{abstract}

\section{Introduction}

The application of scientific and technical achievements, based on the study of physical processes, phenomena, methods, forms and types of their interactions, determines and causes the development of scientific and technical progress in the field of material production. This progress leads to the creation of new technological principles, more efficient technologies, machines, equipment, as well as the improvement and development of existing and new production methods for products. [1] Increasing productivity by higher cutting speed and achieving high precision of machined products at the same time is an important trend in the development of manufacturing technologies and metalworking machine tools. If the traditional method of exchangeable components in assemblies will lead to inefficient precise processes, the product costs will increase.

The development of high-speed (HSC) and highperformance (HPC) cutting technologies has led to the need for both their fundamental research and various studies of real manufacturing processes. So, the impact of various parameters for speed, depth of cut, feed, properties of the processed material, the properties of the machine tool, etc. on the characteristics of the HSC, HPC processes, such as power, energy, temperature, force, speed, vibration, etc. has been evaluated [2,3,4].

A lot of work $[5,6,7]$ (see table 1) is dedicated to the theoretical methods of determining the most important parameters in metal working processes, aiming at the justification of the cutting process models. These models allow describing the physical laws of the process, its parameters, power, thermal and energy characteristics, observed types of chips, tool wear, etc.

Table 1. Cutting models and schemes

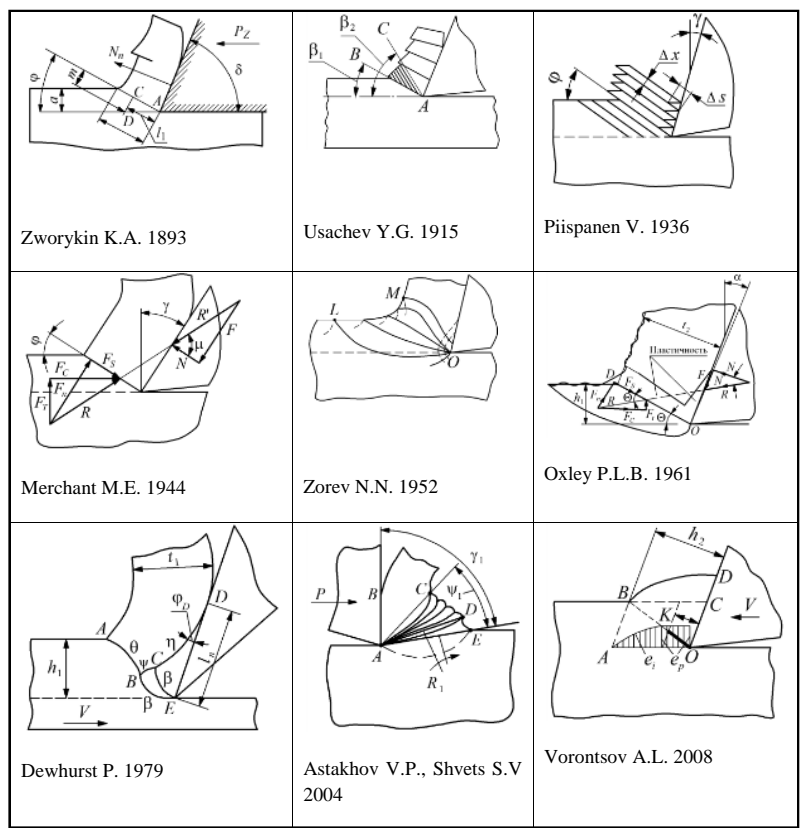

The considered mechanical principles of cutting schemes, their features and models are essential for consideration and creation of cutting processes structures. They reflect not only the physical phenomenon, but also the constituent elements, that determine the possibility of the physical process itself. $[8,9,10]$ In this case, the structure elements of the cutting process will be position 
and motion vectors (velocity and force vectors), which in all cases provide the stress-strain state required for the cutting process. Considering process models and experimental studies, the recommended areas (Fig. 1) for the values of cutting speeds of cutting methods and materials to be processed were determined [11].

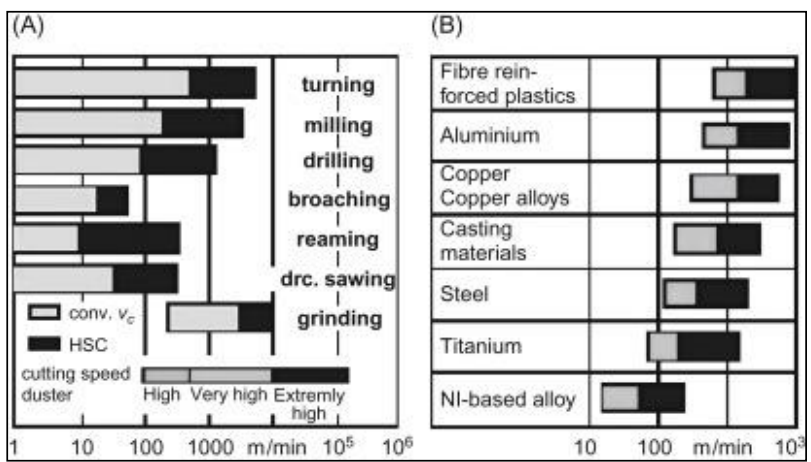

Fig. 1. HSC speed ranges for (a) cutting methods and (b) materials to be processed [11]

However, these values still require a deeper substantiation of the boundaries, the allowable areas and their relationship with the physical metal cutting processes and phenomena.

\section{Energy-informational model}

The work piece material undergoes changes, interacting with the tool. The character and kind of these changes depend on the initial condition, on the structure and properties of the work piece material, how quantitatively and qualitatively it relates with the condition, structure and properties of the tool material. Next, they depend on the supplied energy, in what order, in what quantity and what kind. Further it is important, what quantity of information the tool comprises on a product shape. The material of the tool can also be absent, and then there is an interaction of the work piece material with the energy from the technological environment [1].

The kind of energy for the realised physical process is one of the basic characteristics influencing the interaction between the materials of the work piece and the tool. Usually, the interaction type defines the name of the realized manufacturing method and technological process. The form of energy can be mechanical, thermal, electric, electromagnetic, laser, plasma, chemical, gravitational, etc., as well as their various combinations.

As the purpose of the energy impact is a change in the state and/or structure and/or properties, all these processes are accompanied by real physical phenomena: elastic deformation, plastic deformation, diffusion, evaporation, etc. Plastic deformation depends on how and in what order forces were applied to such technological processes as cutting, punching, forging, and drawing. Thus, the form diversity of interactions between matter, energy and information determines numerous types of technological processes and equipment implementing these technologies.

Fig. 2 presents the energy - information model of the part production $[12,13,14]$. In case of the cutting process, the system components provide the formation of the work piece size. The physical process of plastic deformation is carried out between the tool and the shape and surface of the work piece. The operating elements of the machine tool perform the physical process of surface shaping. Other characteristics and parameters (marked as properties, such as roughness, structure, state, such as hardness, etc.), which describe the information image of the part, are formed by other processing methods in accordance with the technological image of the part and the energy - information model in fig. 2.

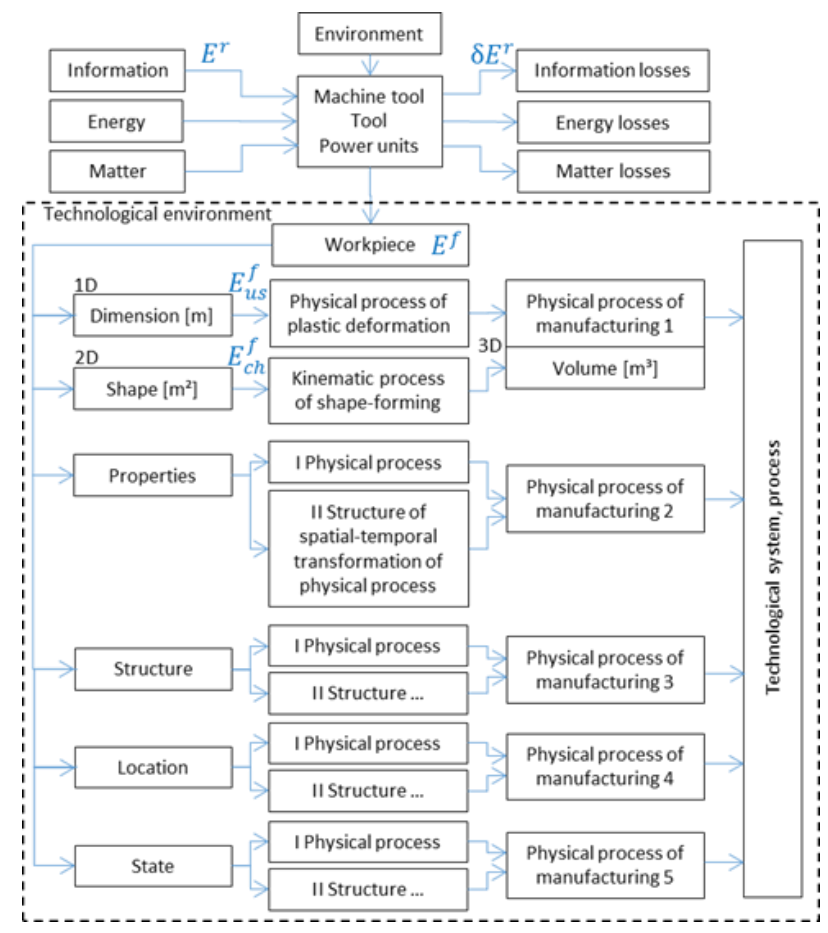

Fig. 2. Energy-informational model [12]

Consequently, the manufacturing process consists of two elements: a set of items of physical processes I (the physical process of plastic deformation, which defines the size, is shown) and its corresponding set of structures of spatial temporal transformation II of the physical process (kinematic process of contouring, which defines the desired shape of the part, is shown). [11]

The proposed energy-informational model in fig. 2 considers a procedural system establishing the ratio of the cutting speed and the speed of physical processes in the machine tools. System elements, ensuring the cutting of part dimension (physical process, implemented by the tool) and the creation of part surfaces (physical process of surface shaping, implemented by operating elements of the machine), are highlighted here. Technological process is performed through the manufacturing equipment with use of material, energy, information. The result of the technological process is the product, which shall meet the customer's expectations.

\subsection{Cutting geometry model}

Design principles of cutting schemes shown in table 1, their features and models are essential for the 
consideration and creation of cutting process structures, considering not the physical phenomenon, but only the constituent elements, that determine a possible implementation of the physical process in accordance with the energy-information model (fig. 2). In this case, the structure elements of the cutting process will be position and motion vectors (velocity and force vectors), which in all cases provide the stress-strain state required for the cutting process.

Linear fracture mechanics describes the brittle fracture resulting from crack growth with little (or no) plastic deformation at the top of the crack. If the characteristic linear size of the plastic zone at the top of the crack exceeds the length of the crack by more than $20 \%$, then the cracked body behaviour is described by nonlinear fracture mechanics characterized by a sufficiently developed plastic zone in front of the top of the crack. Nonlinear fracture mechanics allowed drawing a conclusion that as plastic deformations develop their gradients at the top of the cracks and the shape of plastic zones change. At the same time, elasto-plastic deformations and the size of plastic zones grow disproportionately to the nominal stresses.

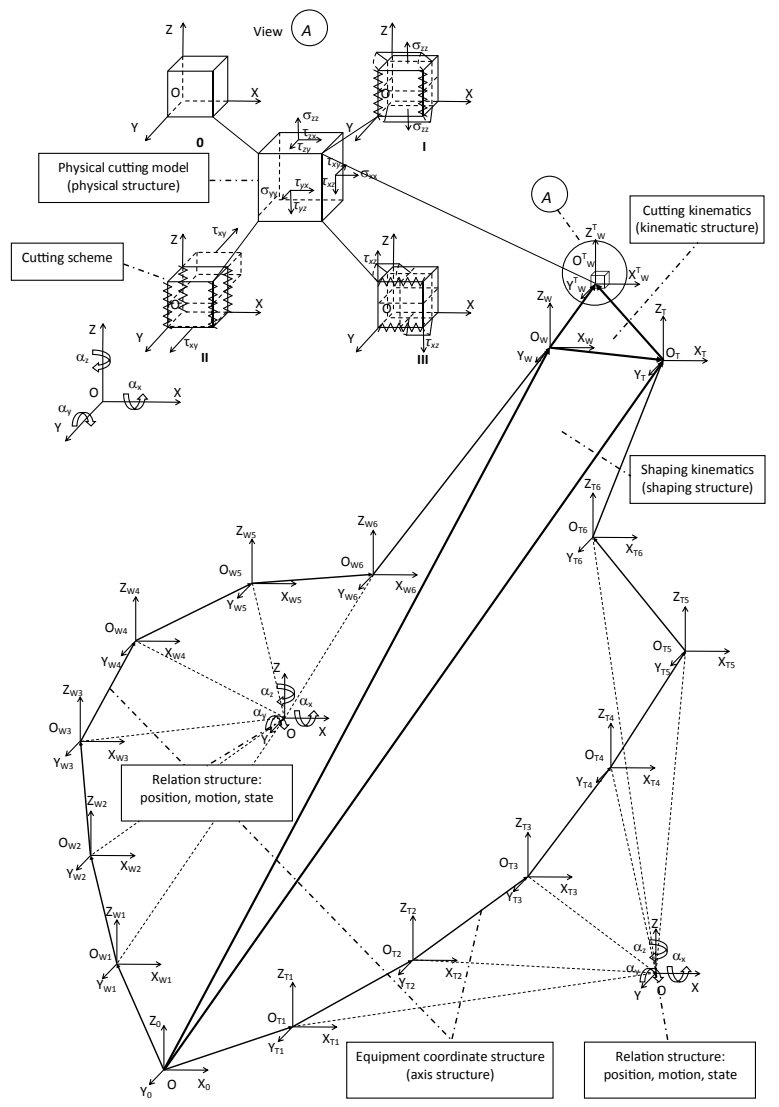

Fig. 3. Process and equipment model for material machining by cutting

Fig. 3 shows the process and equipment model for material machining by cutting in a plane perpendicular to the front of the crack propagation. Here we agree the structural condition for the coordinate systems in this model, stating that the direction of axis $0 Z$ is always perpendicular to a motion plane or a connection plane, and for physical cutting model - to a dislocation plane. Fig. 3 encloses multiple coordinate systems " $\mathrm{O}$ " using different indices $\quad O_{0}, O_{T 1}, \ldots, O_{T 6}, O_{W 1}, \ldots, O_{W 6}, O^{T}, O^{W}, O_{W}^{T}$, where index " 0 " (down) corresponds to an independent general coordinate system, the indexes "T1 ... T6" (right) correspond to coordinate systems that are related to possible positions and movements of the tool. The indexes "W1 ... W6" (left) correspond to coordinate systems that are relating to possible positions and movements of the work piece. The indices "T" or "W" correspond to the systems of fixing points of the tool and the work piece respectively. The index "T-W" corresponds to the point of interaction between the tool and the work piece, representing the location of the physical cutting process.

Connecting structures determine mutual position and motion of coordinate systems in the structure. Their state determines the change of position and/or motion of radius vectors between the centres of $\left(O_{T}-O_{T-1}\right)$ coordinate systems in their state space (spatial, power, thermal, gravitational, time). Each rectangular coordinate system provides three linear movements along the coordinate axes and three rotational movements around the coordinate axes, i.e. six movements that correspond to the degrees of freedom of a solid body. Six position components, three linear components in the axes direction and three angular components of rotation around the axes, determine the relative position.

Thus, the process and equipment model for material machining by cutting on fig. 3 shows the possible number of coordinate systems, each of which is characterized by only one degree of freedom for movement or rotation, while the relative position of the two systems is characterized and determined by six parameters - three linear and three rotational.

Then, in the "A" coordinate system $O_{W}^{T}$ determines the point (line, surface) of interaction between solids (work piece and tool) and determines the coordinate of the position of the stress tensor at the top of the crack. At the point of contact $O_{W}^{T}$ it is also necessary to ensure equality of rectangle and tangent surfaces of contacting solids (work piece and tools), velocity vectors of the work piece and tool, their first and second derivatives.

Figure 3 also shows that:

- the model type for the fracture scheme determines the physical process structure, i.e. the physical cutting model (physical process of plastic deformation and fracture);

- the difference vector of the position module $\left(O_{0}-O_{W}\right)$ and $\left(O_{0}-O_{T}\right)$ with vertices at the points $O_{T}$ and $O_{W}$ defines the volume of working space;

- geometrical and space relations of mutual positions and dimensions of coordinate vectors $\left(O_{W}-O_{W}^{T}\right)$ and $\left(O_{T}-O_{W}^{T}\right)$ determine the structure of the cutting scheme (space position in the model of the physical cutting process);

- change of mutual relative vector movements $\left(O_{W}-O_{W}^{T}\right),\left(O_{W}^{T}-O_{T}\right)$ and $\left(O_{T}-O_{W}\right)$ define schemes of kinematic structures, i.e. the cutting kinematics;

- change of mutual relative vector movements $\left(O_{0}-O_{W}\right)$ and $\left(O_{0}-O_{T}\right)$ defines the schemes of coordinate structures, which implement the 
corresponding schemes of kinematic structures, i.e. the layout of the equipment structures.

Then the operator of $L_{P}$ transformation in homogeneous coordinates will be written:

$M_{W}^{T}=M_{T}^{P} \times M_{T}^{M} \times M_{T 6}^{P} \times M_{T 6}^{M} \times \ldots \times M_{T 1}^{P} \times M_{T 1}^{M} \times$

$M_{0}^{P} \times M_{W 1}^{P} \times M_{W 1}^{M} \times \ldots \times M_{W 6}^{P} \times M_{W 6}^{M} \times M_{T}=L_{P} \times M_{T}$ (1)

where - $M_{W}^{T}$ is the matrix of the moving object (point, line...). $M_{T i}^{P}$ is the location matrix of coordinate position systems and is equal to:

$M_{T i, W}^{P}=\left|\begin{array}{cccc}\cos \alpha_{x x} & \cos \alpha_{x y} & \cos \alpha_{x z} & a_{x} \\ \cos \beta_{y x} & \cos \beta_{z y} & \cos \beta_{y z} & a_{y} \\ \cos \gamma_{z x} & \cos \gamma_{z y} & \cos \gamma_{z z} & a_{z} \\ 0 & 0 & 0 & 1\end{array}\right|$

\subsection{Cutting motion model}

If the coordinate systems are relating to moving elements, the generalized motion matrix will look like:

$M_{W i}^{M}=\left|\begin{array}{cccc}\propto_{11} & \propto_{12} & \propto_{13} & l_{i} \times \cos \varepsilon_{i} \\ \propto_{21} & \propto_{22} & \propto_{23} & l_{i} \times \cos \varphi_{i} \\ \propto_{31} & \propto_{32} & \propto_{33} & l_{i} \times \cos \xi_{i} \\ 0 & 0 & 0 & 1\end{array}\right|$

where $\propto_{i i}$ is a function of the rotational motion of the coordinate system; $l_{i}$ - the value of movement; $l_{i} \times \cos \varepsilon_{i}$, $l_{i} \times \cos \varphi_{i}, l_{i} \times \cos \xi_{i}$ - the guiding cosines of the motion direction. In general, $\propto_{i i}$ and $l_{i}$ are functions that depend on time.

When the coordinate systems are orthogonal and noncrossing, the sequence of rotations of corresponding rotation matrixes around the coordinate axes of $O X, O Y$, and $O Z$ can describe and determine the positions of the coordinate systems. The product of three rotation matrices, each of which describes the rotation motion around one of the coordinate axes, will determine the overall position matrix:

$M_{T, W}^{P}=M_{i, W, T, x}^{P, M} \times M_{i, W, T, y}^{P, M} \times M_{i, W, T, z}^{P, M}$

$M_{i, W, T, x}^{P, M}=\left|\begin{array}{cccc}1 & 0 & 0 & a_{x} \\ 0 & \cos \alpha_{x} & -\sin \alpha_{x} & a_{y} \\ 0 & \sin \alpha_{x} & \cos \alpha_{x} & a_{z} \\ 0 & 0 & 0 & 1\end{array}\right|$

$M_{i, W, T, y}^{P, M}=\left|\begin{array}{cccc}\cos \alpha_{y} & 0 & -\sin \alpha_{y} & a_{x} \\ 0 & 1 & 0 & a_{y} \\ \sin \alpha_{y} & 0 & \cos \alpha_{y} & a_{z} \\ 0 & 0 & 0 & 1\end{array}\right|$

$M_{i, W, T, z}^{P, M}=\left|\begin{array}{cccc}\cos \alpha_{z} & -\sin \alpha_{z} & 0 & a_{x} \\ \sin \alpha_{z} & \cos \alpha_{z} & 0 & a_{y} \\ 0 & 0 & 1 & a_{z} \\ 0 & 0 & 0 & 1\end{array}\right|$

Substituting them in the expression $M_{W i}^{M}$, we get a motion matrix that determines the given motion law.

Coordinates of the point $O_{W}^{T}$ in the independent general coordinate system $\left(O X_{0} Y_{0} Z_{0}\right)$ will be written: $M_{W}^{0}=M_{W}^{P} \times M_{W}^{M} \times M_{W 6}^{P} \times M_{W 6}^{M} \times \ldots \times M_{W 1}^{P} \times M_{W 1}^{M} \times$ $M_{W}$
$M_{T}^{0}=M_{T}^{P} \times M_{T}^{M} \times M_{T 6}^{P} \times M_{T 6}^{M} \times \ldots \times M_{T 1}^{P} \times M_{T 1}^{M} \times M_{T}$

Independent from the chosen method of formation, the number and sequence of coordinate systems types, the equality condition of coordinates in the point $O_{W}^{T}$ must always be observed, i.e. equality: $M_{W}^{0}=M_{T}^{0}$.
By entering the generalized velocity coordinate, dividing the expression by the time "t" in homogeneous coordinates, we get the generalized velocity structure of the model:

$\bar{M}_{W}^{T}=M_{T}^{P} \times \bar{M}_{T}^{M} \times M_{T 6}^{P} \times \bar{M}_{T 6}^{M} \times \ldots \times M_{T 1}^{P} \times \bar{M}_{T 1}^{M} \times$

$M_{0}^{P} \times M_{W 1}^{P} \times \bar{M}_{W 1}^{M} \times \ldots \times M_{W 6}^{P} \times \bar{M}_{W 6}^{M} \times M_{T}$

\subsection{Cutting process model}

For the cutting process, the model of the fracture scheme type determines the physical structure of the process, i.e. the physical cutting model (the physical process of plastic deformation and fracture). In our case, the stress tensor, as described above for different cutting schemes in the $Z O X$ plane perpendicular to the crack front, is determined by the flat stress state, for the dislocation of the shift in the $X O Y$ plane, in the form of:

$T_{\sigma}=\left(\begin{array}{ccc}\sigma_{x x} & 0 & \tau_{x z} \\ 0 & 0 & 0 \\ -\tau_{z x} & 0 & \sigma_{z z}\end{array}\right)$

Similar to the shear stress tensor $\bar{\tau}_{S}=\tau_{z x}$, the speed (velocity tensor) $\bar{v}_{S}=v_{z x}$ and its components will be equal to:

$v_{z x}\left(\tau_{z x}\right)=v_{z x}+v_{y x}+R \times \omega_{y}=v_{y x}+v_{z x}+R_{z 0} \times$

$\omega_{y 0}^{z}+R_{y 0} \times \omega_{y 0}^{y}+R_{x 0} \times \omega_{y 0}^{x}$

where $v_{z x}, v_{y x}$ is the linear velocity vector of the axes

$\mathrm{Z}_{0}, \mathrm{Y}_{0}$ along $\mathrm{X}_{0}$ in the shear plane $X O Y$.

$R \times \omega_{y}$ is the linear vector of the rotation speed of the radius-vector around the axes in the $X O Y$ shear plane.

Tangential stresses $\tau_{S}$ determining the dislocation shift are equal to:

$\tau_{S}=\tau_{x y}=\frac{F_{S}}{A_{S}}$

where $F_{S}$ is the shear force of the dislocation, $A_{S}$ is the tangential stress area.

The shear occurs when the tangential stresses and active forces on the part and tool side are equal (friction forces are not considered here), as a result we will get: $\bar{F}_{S}=\bar{\tau}_{S} \times A_{S}=m_{S} \times a_{S}=\rho \times V_{S} \times \frac{\bar{v}_{S}}{t_{S}}=\sum \bar{F}_{T W}=$ $m_{W} \times a_{W}+m_{T} \times a_{T}=m_{W} \times \frac{\bar{v}_{W}}{t}+m_{T} \times \frac{\bar{v}_{T}}{t}$

where $a_{S}$ is the acceleration and $\bar{v}_{S}$ is the dislocation speed vector, $t_{S}$ is the dislocation propagation time, $V_{S}$ the moved dislocation volume, $m_{W}$ - the work piece mass, $m_{T}$ - the tool mass, $\bar{v}_{W}$ - the vector of the work piece speed, $\bar{v}_{T}$ - the vector of the tool speed, $\mathrm{t}$ - the cutting time.

A more precise value of the active force during the cutting process can be found in the works of different authors, whose cutting schemes are shown in table 1 .

In this case, the structure of the cutting force is important, which can be written as:

$\bar{F}_{S}=\bar{\tau}_{S} \times K_{S}$

where the coefficient $K_{S}$ is determined by the cutting model under consideration (table 1) and depends on the type and quantity of parameters taken into account (geometric linear and angular, physical properties, e.g. friction type, etc.) and the degree of their detailing.

For example, in the Vorontsov A.L. scheme 2008 the cutting force is determined as follows: 


$$
\begin{aligned}
& F_{S}=\tau_{S} \times K_{S}=\beta \times \sigma_{S} \times u \times h_{1} \times\left\{\cos \gamma \times\left[1+\frac{\mu_{1} \times l}{h_{2}}+\right.\right. \\
& \left.\left.\frac{(0.5+\mu) \times u \times h_{1}}{2 \times h_{2}}\right]+\frac{h_{2}}{4 \times u \times h_{1} \times \cos \gamma}+\mu \times \sin \gamma+\frac{\mu_{2} \times l_{3}}{u \times h_{1}}\right\} \\
& \tau_{x y}=\tau_{S}=\beta \times \sigma_{S} \times\left(\mu-\frac{0.5+\mu}{h_{2}} \times y\right) \\
& \tau_{S} \times K_{S}=\beta \times \sigma_{S} \times\left(\mu-\frac{0.5+\mu}{h_{2}} \times y\right) \times K_{S}=\beta \times \sigma_{S} \times \\
& u \times h_{1} \times\left\{\cos \gamma \times\left[1+\frac{\mu_{1} \times l}{h_{2}}+\frac{(0.5+\mu) \times u \times h_{1}}{2 \times h_{2}}\right]+\right. \\
& \left.\frac{h_{2}}{4 \times u \times h_{1} \times \cos \gamma}+\mu \times \sin \gamma+\frac{\mu_{2} \times l_{3}}{u \times h_{1}}\right\}
\end{aligned}
$$

Therefore, the coefficient $K_{S}$ in the case of Vorontsov A.L. scheme 2008 is equal to:

$$
\begin{aligned}
& K_{S}=\left(\mu-\frac{0.5+\mu}{h_{2}} \times y\right)^{-1} \times u \times h_{1} \times\{\cos \gamma \times[1+ \\
& \left.\left.\frac{\mu_{1} \times l}{h_{2}}+\frac{(0.5+\mu) \times u \times h_{1}}{2 \times h_{2}}\right]+\frac{h_{2}}{4 \times u \times h_{1} \times \cos \gamma}+\mu \times \sin \gamma+\frac{\mu_{2} \times l_{3}}{u \times h_{1}}\right\}
\end{aligned}
$$

For other cutting schemes in Table 1, as well as the existing schemes of other authors, the cutting force will be determined similarly to

$F_{S}=\tau_{S} \times K_{S}$, but the values of $K_{S}$ will differ numerically, the value of which is determined by the values of angles in the adopted schemes, the values of friction coefficients. At the same time, the value of shear stresses in the most general form is determined from the ratio:

$$
\begin{gathered}
\tau_{S}=\frac{\sigma}{\sqrt{3}} \\
\text { where } \sigma \text { according to the Johnson-Cook equation } \\
\bar{\sigma}=\left[A+B(\varepsilon)^{n}\right] \times\left[1+C \times \ln \left(\frac{\bar{\varepsilon}}{\bar{\varepsilon}_{0}}\right)\right] \times\left[1-\left(\frac{T-T_{0}}{T_{m}-T_{0}}\right)^{m}\right]
\end{gathered}
$$

Due to space limitations, this material does not include a table of $K_{S}$ values for the thirty most well known cutting models.

\section{Energy limit}

\subsection{Allowable manufacturing process speed}

The energetic limit for the attainability of associated processes describes the allowable speed of materials processing by the machine tools. Significant advances in understanding the machining process were made by Ernst and Merchant, whose thermodynamic approach proposed a specific cutting energy Ps, which describes the efficiency of the cutting process:

$P_{S}=\frac{P_{m}}{Z_{w}}=\frac{F_{c}}{A_{c}}$

Here $P_{m}=F_{c} \times v$ is the rate of energy consumption during machining; $F_{c}$ - cutting force in the direction of cutting; $\mathrm{Z}_{\mathrm{w}}$ - metal removal rate; $\mathrm{A}_{\mathrm{c}}$ - cross-sectional area of the undeformed chip, and $\mathrm{v}$ - cutting speed.

$\mathrm{P}_{\mathrm{S}}$ is affected by cutting speed, feed and tool geometry. The theory does not specifically provide for materials comparisons in that Ps can vary considerably for a particular material. Cutting energy does tend to become constant at high speeds and large feeds, therefore Ernst and Merchant, and Lee and Shaffer later on, imply that the dominant material property is related to the work piece material's shear strength. (Experimental work does in fact show that a material's shear stress remains constant over a wide range of cutting conditions.) Thermodynamics also suggests that the non-friction heat generated during machining is a function of the work piece material's physical properties. One proposal is that it should therefore be possible to describe machinability in terms of a "thermal number" $\mathrm{R}$, which considers the relevant properties:

$\mathrm{R}=\frac{\rho \times c \times v \times a}{k}$

where $\rho$ - density, c - heat capacity, $\mathrm{v}$ - velocity related to cutting speed, a - chip area, $\mathrm{k}$ - work piece thermal conductivity. [15]

The product of the force and velocity vector are used to predict power requirements for sizing a new machine tool or for predicting the production rates possible with an existing machine tool. Temperature predictions are used to estimate how the process of machining or grinding affects a useful life of cutting edge or how elevated temperatures can change the mechanical properties of the work piece. [11]

The main body of the ISO 3685-2017 report is contained in several sections which deal with reference work piece materials, reference tool materials and tool geometries, reference cutting fluids, reference cutting conditions, tool life criteria and tool wear measurement, equipment, test procedures, and the recording and reporting of results. $[16,17]$

\subsection{Physical cutting process model}

Based on Fig. 1, let's present the energy model of cutting as a physical process of transformation of the initial material (characterized by mass, volume, physical and mechanical properties) into a given part (characterized also by its mass, volume, physical and mechanical properties) under the influence of energy required for this transformation.

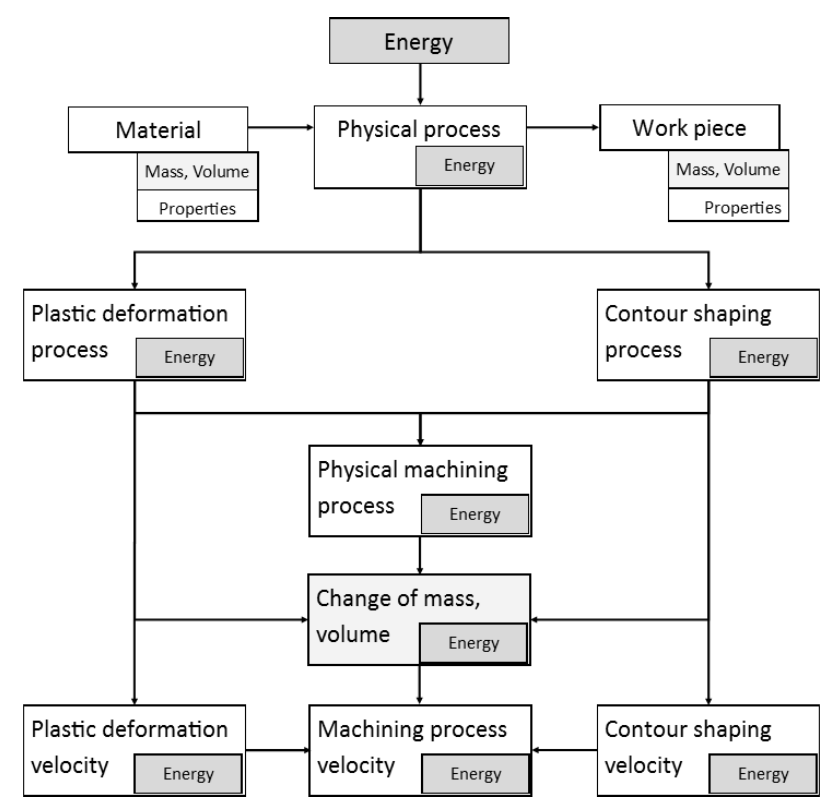

Fig. 4. Energetic model on energy transformation and material property change velocity 
The fracture model determines the physical structure of the process - the physical model of cutting (the physical process of plastic deformation and fracture in Fig. 4), and the shear stresses determining the dislocation shift are equal to:

$\tau_{S}=\tau_{x y}=\frac{F_{S}}{A_{S}}$

The shift occurs when the shear stresses and effective forces on the part and tool side are equal (see Section 2).

In this case, it is important, as shown in section 2 above, that the cutting force structure can be written as: $\bar{F}_{S}=\bar{\tau}_{S} \times K_{S}$

where the coefficient $K_{S}$ is determined by the cutting model under consideration and depends on the type and quantity of parameters taken into account (geometric linear and angular, physical properties, e.g. friction type, etc.) and their degree of detail. At the same time, the cutting force operation is determined by the cutting force and cutting speed.

Consequently, this relation build the following equation:

$\tau_{S}=\frac{F}{A_{S}}=\frac{F \times L}{A_{S} \times L}=\frac{F \times L}{A_{S} \times L} \times \frac{\tau}{\tau}=\frac{F \times v}{\frac{V}{\tau}}=\frac{P}{Q}=\frac{W}{V}$

where $\mathrm{F}$ is force $[\mathrm{N}]$; AS is area $\left[\mathrm{m}^{2}\right]$; $\mathrm{L}$ is length (cutting motion) $[\mathrm{m}] ; \tau$ is time $[\mathrm{s}(\mathrm{min})]$; $\mathrm{V}$ is volume $\left[\mathrm{m}^{3}\right]$; $v$ is speed $[\mathrm{m} / \mathrm{s}, \mathrm{m} / \mathrm{min}]$; $\mathrm{P}$ is power $[\mathrm{W}]$; $\mathrm{Q}$ is specific productivity $\left[\mathrm{m}^{3} / \mathrm{s}\right] ; \mathrm{W}$ is energy, $\mathrm{J}$ is specific energy intensity $\left[\mathrm{J} / \mathrm{m}^{3}\right]$.

This expression defines various stress ratios, including the value of shear stresses and relevant $\sigma_{s}$ - yield stress of the processed material. From this conceptual dependence follows the values of energy ranges, required for cutting of different materials under different cutting conditions (Table 2).

Table 2. Values of energy ranges, required for cutting of different materials under different cutting conditions [12]

\begin{tabular}{|c|c|c|c|}
\hline $\begin{array}{l}\text { Chip removal } \\
\text { range }\end{array}$ & $\begin{array}{l}1 \mathrm{~nm} \ldots \\
0.1 \mu \mathrm{m}\end{array}$ & $\begin{array}{c}0.1 \mu \mathrm{m} \ldots \\
10 \mu \mathrm{m}\end{array}$ & $\begin{array}{c}10 \mu \mathrm{m} \ldots \\
1 \mathrm{~mm}\end{array}$ \\
\hline $\begin{array}{l}\text { Defects / } \\
\text { Inserts }\end{array}$ & Spot defect & $\begin{array}{l}\text { Offset/ } \\
\text { Crack }\end{array}$ & $\begin{array}{c}\text { Crack / } \\
\text { crystal border }\end{array}$ \\
\hline $\begin{array}{c}\text { Type of } \\
\text { material } \\
\text { destruction } \\
\end{array}$ & $\begin{array}{l}\text { atomic } \\
\text { cluster }\end{array}$ & $\begin{array}{c}\text { Sub } \\
\text { crystalline }\end{array}$ & $\begin{array}{c}\text { Multi } \\
\text { crystalline }\end{array}$ \\
\hline $\begin{array}{c}\text { Resistance to } \\
\text { brittle } \\
\text { fracture }\end{array}$ & $\begin{array}{c}10^{4} \ldots 10^{3} \\
\mathrm{~J} / \mathrm{m}^{3} \\
\text { atomic } \\
\text { crack }\end{array}$ & $\begin{array}{c}10^{3} \ldots 10^{2} \\
\mathrm{~J} / \mathrm{m}^{3} \\
\text { micro crack }\end{array}$ & $\begin{array}{c}10^{2} \ldots 10 \\
\mathrm{~J} / \mathrm{m}^{3} \\
\text { fragile crack }\end{array}$ \\
\hline $\begin{array}{l}\text { Shear } \\
\text { resistance } \\
\text { limit }\end{array}$ & $\begin{array}{c}10^{4} \ldots 10^{3} \\
\mathrm{~J} / \mathrm{m}^{3} \\
\text { atomic dis- } \\
\text { placement }\end{array}$ & $\begin{array}{c}10^{3} \ldots 10^{2} \\
\mathrm{~J} / \mathrm{m}^{3} \\
\text { slip offset }\end{array}$ & $\begin{array}{c}10^{2} \ldots 10 \\
\mathrm{~J} / \mathrm{m}^{3} \\
\text { shear strain }\end{array}$ \\
\hline
\end{tabular}

\subsection{Physical crack propagation speed}

The physical cutting process (Fig. 4) determines and origins the material removal speed during cutting and is characterized by the speed of relative movement of the cutting tool and work piece, which can be determined by the product of the area of material cut per unit of time or the feed rate or cutting speed multiplied by the cutting area. Thus, you can write:
$Q=\frac{F \times v}{\tau_{s}}=\frac{\tau_{s} \times K_{S} \times v}{\tau_{s}}=K_{s} \times v$

The ideal rate of propagation of plastic deformations can be determined based on the solution of the equation of motion of the mass element of the material having the form:

$\frac{\partial^{2} u}{\partial t^{2}}=\frac{\partial \sigma}{\partial x}=\frac{E}{\rho} \times \frac{\partial^{2} u}{\partial t^{2}}$

Then the elastic wave propagation velocity

$v=\sqrt{\frac{E}{\rho}}$

can be taken as a measure of the plastic deformation propagation velocity.

Therefore, if we take into account the recommendations of [12] about the dependence of the plastic strain rate on the sound velocity:

$v_{\text {dislocation }}=\sqrt{\frac{E}{\rho}} \times e^{-\frac{A}{\left(\tau_{S} \times \theta^{0}\right)}}=v_{\text {sound }} \times e^{-\frac{A}{\left(\tau_{S} \times \theta^{0}\right)}}$

where $\mathrm{A}$ is the material constant, $\tau_{\mathrm{s}}$ is the shear stress, $\theta^{0}$ is the cutting zone temperature.

Table 3. Sound and crack velocities for various materials [18]

\begin{tabular}{|c|c|c|}
\hline Material & $\begin{array}{c}\text { Sound velocity } \\
{[\mathrm{m} / \mathrm{s}]}\end{array}$ & $\begin{array}{c}\text { Crack propagation } \\
\text { velocity }[\mathrm{m} / \mathrm{s}]\end{array}$ \\
\hline Diamond & 12000 & $6000-7200$ \\
\hline Steel & 5030 & 1800 \\
\hline Hardened steel & 5850 & 2300 \\
\hline
\end{tabular}

The scheme of formation and direction of crack propagation is shown in Fig.5.

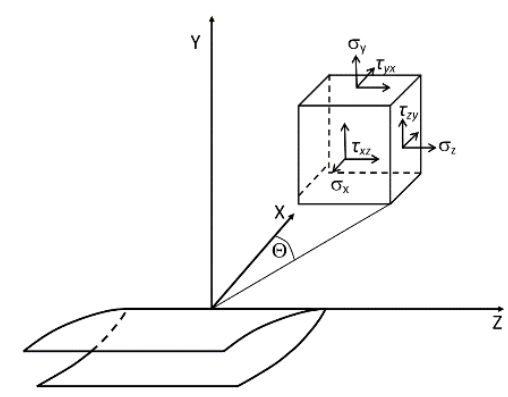

Fig. 5. Stress components and crack propagation

The driving force behind the crack propagation is the energy of deformation, which is balanced by surface and kinetic energies, and as the velocity of the crack increases above the maximum velocity, it will be absolutely brittle. It is also known that the speed of heat waves propagation is determined by the speed of sound. Therefore, at supersonic speeds there is a "lagging behind" of the heat flow and, consequently, a decrease in the coefficient of thermal conductivity of metals with an increase in processing speed.

Taking into account the kinetic energy of the crack, the maximum possible rate of propagation of the fracture in the solid body, referring to Mott, is 0.38 of the sound velocity, and within the framework of a purely elastic problem, this limit is increased to 0.4 of the sound velocity. The above arguments about velocity at plastic deformation and fracture allow us to raise the question 
about the maximum possible cutting velocity. If we assume that the thickness of the material to be cut $h$, the direction of propagation is determined by the angle $\theta$, the length of the crack will be equal to:

$l_{\text {crack }}=\frac{h}{\sin \theta}$

The propagation of this crack will require a time equal to:

$$
t_{\text {crack }}=\frac{h}{\sin \theta \times \mathrm{v}_{\text {dislocation }}}=\frac{h}{\sin \theta \times \sqrt{\frac{E}{\rho} \times e^{-\frac{A}{\left(\tau_{s} \times \theta^{0}\right)}}}}
$$

The length projection is cracked on the cutting speed direction will be equal:

$$
L_{\text {crack }}=l_{\text {crack }} \times \cos \theta=\frac{h \times \cos \theta}{\sin \theta}
$$

The time required to travel this distance will be equal to:

$$
t_{\text {crack }}=\frac{L_{\text {crack }}}{v_{\text {crack }}}=\frac{h \times \cos \theta}{\sin \theta \times v_{\text {crack }}}
$$

Then for the process of formation and removal of dislocations to be continuous it is necessary to have equality of times

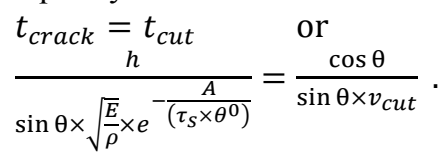

Therefore, the cutting speed will be determined as follows:

$v_{\text {cut }}=\frac{\cos \theta \times h \times \sin \theta}{\sin \theta \times h} \times \sqrt{\frac{E}{\rho}} \times e^{-\frac{A}{\left(\tau_{S} \times \theta^{0}\right)}}=\cos \theta \times \sqrt{\frac{E}{\rho}} \times$

$e^{-\frac{A}{\left(\tau_{S} \times \theta^{0}\right)}}=\cos \theta \times v_{\text {sound }} \times e^{-\frac{A}{\left(\tau_{S} \times \theta^{0}\right)}}$.

Therefore, in accordance with the scheme of Fig. 4, the forming rates and plastic deformation rates differ and depend on the direction of propagation are cracked, and the kinematically necessary cutting rate is proportional to the rate of propagation of plastic deformations multiplied by the cosine of the angle of propagation of dislocations.

Energy parameters of each of the components of the cutting structure are determined by the above dependencies based on the required velocity ratios. On this basis, the question of requirements to the physical characteristics of the machines designed for this purpose is solved.

\section{Increasing productivity}

\subsection{Stiffness of machine tool structures}

Stiffness of machine tool structures [3] for highest precision manufacturing becomes comparable to work piece geometrical accuracy and manufacturing process tolerances.

\subsection{Traditional cutting application}

The cutting velocity for a steel work piece by a standard milling tool is $v_{c}=1 \mathrm{~m} / \mathrm{s}$. The cutting force is $F_{c}=3000 \mathrm{~N}$, generated by an electrical servo drive and ball screw. The rate of energy consumption during machining is

$P_{m}=F_{c} \times v_{c}=3000 \mathrm{Nm} / \mathrm{s}$.
The cutting time is $300 \mathrm{~s}$. The cutting energy consumption is $900000 \mathrm{~J}$. The axial work piece accuracy is $\mathrm{P}=0.01 \mathrm{~mm}$. The required machine tool stiffness is $c=\frac{F_{C}}{P}=\frac{3000 \mathrm{~N}}{0.01 \mathrm{~mm}}=300000000 \mathrm{~N} / \mathrm{m}$.

\subsection{High Speed Cutting application}

The cutting velocity for a steel work piece by a HSC milling tool is $v_{c}=25 \mathrm{~m} / \mathrm{s}$. The cutting force is $F_{c}=2000 \mathrm{~N}$, generated by an electrical servo drive and ball screw. The rate of energy consumption during machining is

$P_{m}=F_{c} \times v_{c}=50000 \mathrm{Nm} / \mathrm{s}$

The cutting time is $15 \mathrm{~s}$. The cutting energy consumption is $750000 \mathrm{~J}$. The axial work piece accuracy is $\mathrm{P}=0.01 \mathrm{~mm}$. The required machine tool stiffness is $c=\frac{F_{C}}{P}=\frac{2000 \mathrm{~N}}{0.01 \mathrm{~mm}}=200000000 \mathrm{~N} / \mathrm{m}$.

Table 4. Ratios between traditional cutting and innovative High Speed Cutting technologies

\begin{tabular}{|l|l|l|l|}
\hline Technologies & $\begin{array}{l}\text { Traditional } \\
\text { cutting }\end{array}$ & HSC cutting & ratio \\
\hline Process time [s] & 300 & 15 & 20 \\
\hline Velocity [m/s] & 1 & 25 & 25 \\
\hline Force $[\mathrm{N}]$ & 3000 & 2000 & 0.67 \\
\hline Energy $[\mathrm{J}]$ & 900000 & 750000 & 0.83 \\
\hline Stiffness $[\mathrm{N} / \mathrm{m}]$ & 300000000 & 200000000 & 0.67 \\
\hline
\end{tabular}

The innovative HSC productivity is 20 times faster than a traditional cutting process. The HSC velocity ratio to cutting is 25 , but the process force ratio is $2 / 3$. At the same time, the energy consumption for the HSC process is $83 \%$, but the required machine tool stiffness is $67 \%$ of traditional cutting machine tool.

\section{Summary}

Today's process velocities are far away from theoretical values for crack propagation velocities in table 3 . Not only the process speed is limiting the interaction between the tool and the work piece, but also the energy, the process temperature, the dynamic stability, the tool lifetime and the machine tool stiffness.

This paper gives several examples for admissible limits of increased productivity by increased process speeds in various manufacturing technologies for both traditional cutting and high speed cutting methods.

The energy - information regularities of increasing productivity in metalworking machine tools, presented in this paper, will open chances for innovative manufacturing methods and technologies.

\section{Acknowledgement}

This work was carried out using equipment provided by Fraunhofer IWU and the Center of Collective Use of MSTU "STANKIN". 


\section{References}

1. A.P. Kuznetsov, H.-J. Koriath, Proc. Manufact. 8, 199-206 (2017)

2. R. Neugebauer, K.-D. Bouzakis, B. Denkena, F. Klocke, A. Sterzing, A.E. Tekkaya, R. Wertheim, CIRP Annals Manufact. Techn. 60, 627-650 (2011)

3. H. Schulz, T. Moriwaki, CIRP Annals 41(2), 637643, (1992)

4. S. Ashley, Mech. Eng. 117(5), 56-61 (1995)

5. V.I. Malyshev, Essays on the history of cutting materials science, 216 (2011)

6. E. Merchant, S. Moehring, An interpretive review of 20th century US machining and grinding research, 146 (2003)

7. V.P. Astakhov, M.O.M. Osman, M.T. Hayajneh, Int. J. of Mach. Tools Manufact. 41(3), 393-418 (2001)

8. S.N. Grigoriev, M.P. Kozochkin, F.S. Sabirov, A.A. Kutin, Proc. CIRP 1, 599-604 (2012)

9. S.N. Grigoriev, V.A. Sinopalnikov, M.V. Tereshin, V.D. Gurin, Measur. Techn. 55(5), 555-558 (2012)

10. S.N. Grigoriev, V.D. Gurin, M.A. Volosova, N. Y. Cherkasova, Materialwiss. Werkstofftech. 44(9), 790-796 (2013)

11. W. De Vries, Mechanics and Thermal Models for Machining, 39-79 (1992)

12. M. Putz, A.P. Kuznetsov, H.-J. Koriath, MM Science Journal (Nov. 2019)

13. U. Götze, H.-J. Koriath, et al., CIRP J. of Manufact. Sc. Techn. 5, 151-163 (2012)

14. A.P. Kuznetsov, P. Blau, H.-J. Koriath, Proc. CIRP 46, 340-343 (2016)

15. E. Thiele, K. Kundig, D. Murphy, G. Soloway B. Duffin, SAE Transactions 99(5), 362-371 (1990)

16. B. Mills, A.H. Redford, Machinability of Engineering Materials (1983)

17. ISO 3685:2017 Tool-life testing with single-point turning tools (2017)

18. A.M. Vulf, Metal cutting, 496 (1973) 